are obtained by other reasonable assumptions. If we take into consideration the fact that the $\mathrm{C}-\mathrm{Cl}$ bond of this molecule has the possibility of larger ionic character, as evidenced by the abnormally larger $\mathrm{C}-\mathrm{Cl}$ distance in propargyl chloride, ${ }^{4}$ the electrostatic potential may be larger than that calculated above and come nearer to the observed value. Thus, we can safely conclude the hindering potential of this molecule is mainly due to the electrostatic interaction of the dipoles in the rotating groups.

It should be mphasized that the moments obtained in benzene solution are almost the same as those in carbon tetrachloride, and, in particular, they show an increase with temperature. This result stands out in sharp contrast to that of dichloroethane, ${ }^{5}$ which indicated an abnormally larger dipole moment in benzene solution. This "benzene effect" was interpreted by the assumption ${ }^{5}$ that their molecules associate in benzene solution with the solvent molecules. As stated above, in dichlorobutine the distance between the two chlorine atoms is larger than the diameter of benzene molecule, so that the special association such as found in dichloroethane may not take place in this case.

The details will be published elsewhere in the near future. We are grateful to Professor 0 . Shimamura for his kindness in preparing the sample of dichlorobutine.

${ }^{1}$ B. L. Crawford, Jr., and W. W. Rice, J. Chem. Phys. 7, 435 (1939); G. B. Kistiakowsky and W. W. Rice, J. Chem. Phys. 8, $618(1940)$; Osborne, Garner, and Yost, J. Chem. Phys. 8, 131 (1940).

2 R. A. Oriani and C. P. Smyth, J. Chem, Phys. 17, 1174 (1949)

W Watanabe, Mizushima, Masiko, Sci. Pap. Inst. Phys. Chem. Research (Tokyo) 40,425 (1943); J. Powling and H. J. Bernstein, J. Am. Chem. Soc, $73,1815(1951)$.

" Pauling, Gordy, and Saylor. J. Am. Chem. Soc, 64, 1753 (1942).

o Mizushima, Morino, and Higashi, Sci. Pap. Inst. Phys. Chem. Research (Tokyo) 25, 159 (1934).

\section{The Effect of Hydrogen Bonding on the Hindered Rotation of the Hydroxyl Group in Alcohols}

A. V. STUart AND G. B. B. M. SUTHERLAND

Plysics Deparment, Unine sity of Mickigan, Ann Arbor, Michigan

(Received September 26, 1952)

$\mathrm{I}$

$T$ has been shown by Dennison and his co-workers ${ }^{1,2}$ that in 1 gaseous methyl alcohol, the OH group executes a hindered rotation (or libration) about the $\mathrm{CO}$ axis and that the height of the potential barrier hindered free rotation is about $380 \mathrm{~cm}^{-1}(1.1$ $\mathrm{kcal} /$ mole). If one regards this motion as a libration, then the value of the corresponding normal frequency is approximately 225 $\mathrm{cm}^{-1}$. However, the motion is far from simple harmonic, and the infrared absorption due to this libration extends from about 700 $\mathrm{cm}^{-1}$ to below $50 \mathrm{~cm}^{-1}$ for isolated $\mathrm{CH}_{3} \mathrm{OH}$ molecules in the gaseous state. When methyl alcohol is in the liquid state, where it is known that hydrogen bonding exists between the $\mathrm{OH}$ groups of

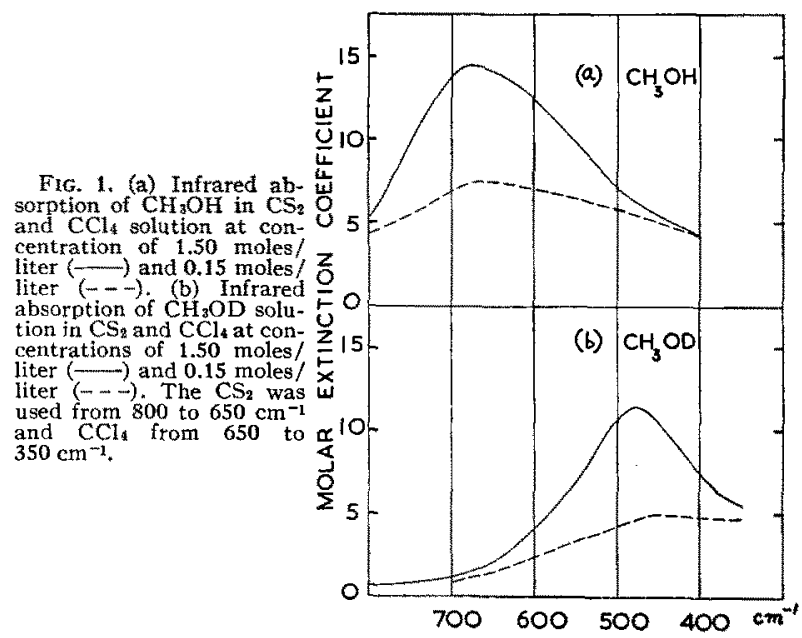

neighboring molecules, it is to be expected that the hindred rotation will be completely inhibited and this motion will now more closely resemble the usual type of fundamental vibration. One would also anticipate that the value of the fundamental frequency would be very much greater than $225 \mathrm{~cm}^{-1}$, since the restoring forces will have increased considerably.

Investigation of the infrared spectra of a series of alcohols reveals a broad absorption band starting about $800 \mathrm{~cm}^{-1}$, reaching a maximum near $670 \mathrm{~cm}^{-1}$ and extending to beyond $500 \mathrm{~cm}^{-1}$. If, by deuteration, the $\mathrm{OH}$ group is converted into an OD group, this band is found to shift to lower frequencies, the maximum now being found close to $475 \mathrm{~cm}^{-1}$ in the case of methyl alcohol (Fig. 1). Since the ratio of the frequencies of maximum absorption is almost exactly $\sqrt{2}$, the $670 \mathrm{~cm}^{-1}$ band must be due to a pure deformation vibration of the $\mathrm{OH}$ group. It seems most probable that it is the deformation vibration in which the hydrogen moves at right angles to the $\mathrm{COH}$ plane, i.e., the motion which in this gaseous state becomes the hindered rotation of the $\mathrm{OH}$ group.

If the hydrogen bonds are now broken up by diluting the alcohol to a low concentration in carbon disulfide or carbon tetrachloride, one would expect the $670 \mathrm{~cm}^{-1}$ band to disappear and be replaced by a band at lower frequencies. The effect of dilution on this band in $\mathrm{CH}_{3} \mathrm{OH}$ and $\mathrm{CH}_{3} \mathrm{OD}$ is also illustrated in Fig. 1. This dilution effect has been checked for a large number of alcohols. It will be seen that the intensity of this band does decrease enormously when the molar concentration is lowered to a point where the degree of hydrogen bonding is known to be low from studies on the $\mathrm{OH}$ stretching frequency near $3250 \mathrm{~cm}^{-1}$. It has not yet been possible to determine where this band moves to on dilution, but studies are being continued in the low frequency region. It is interesting and significant that this $670 \mathrm{~cm}^{-1}$ band has a broad diffuse shape, very similar in extent to the well-known hydrogen-bonded $\mathrm{OH}$ stretching frequency near $3250 \mathrm{~cm}^{-1}$.

The effect of hydrogen bonding on the in-plane $\mathrm{OH}$ deformation vibration has also been studied. The results here are not so straightforward and will be presented in a subsequent publication.

IJ. S. Koehler and D. M. Dennison, Phys. Rev. 57, 1006 (1940). D. C. Burkhard and D. M. Dennison, Phys. Rev. 86, 108 (195i),

\section{Note on the Free Volume Equation of State for Hard Spheres}

\author{
Lous H, LuND \\ Missouri School of Mines and Metallurgy, Rolla, Missouri \\ (Received October 14, 1952)
}

TN a recent Letter to the Editor, Wood ${ }^{1}$ has given an exact $I$ solution for a system composed of rigid spheres to the following integral equation derived by Kirkwood ${ }^{2}$ in his critique of the free volume theory of the liquid state

$$
\begin{aligned}
\varphi(\mathbf{r}) & =\exp \beta[\alpha-\psi(\mathbf{r})], \\
\psi(\mathbf{r}) & =\sum_{1} \int_{c e 1 !} V_{11}\left(\mathbf{R}_{11}+\mathbf{r}-\mathbf{r}^{\prime}\right) \exp \left[\beta\left(\alpha-\psi\left(\mathbf{r}^{\prime}\right)\right)\right] d v^{\prime}, \\
\exp -\beta \alpha & =\int_{c e l 1} \exp -\beta \psi(\mathbf{r}) d v=v_{j},
\end{aligned}
$$

where $\varphi(\mathbf{r})$ is the configurational distribution function for a molecule in its cell, and the terms in $\bar{E}$, the energy with all molecules at their cell centers, are omitted since it is here zero.

Wood has considered a face-centered cubic lattice and chosen as the cell the dodecahedron $\Delta$ of Buehler $e t a l .^{3}$ The nearest neighbor distance $a$ and volume per molecule $v$ are related by $a^{3}=\sqrt{ } 2(v)$, and the minimum volume per molecules is $v_{0}=r_{0}^{3} / \sqrt{ } 2$, where $r_{0}$ denotes the diameter of the sphere. His solution for the system of $\mathrm{Eq}$. (1) is

$$
\psi(\mathbf{r})=\left\{\begin{array}{l}
0 \text { if } r \text { is in } \Delta^{\prime} \\
\infty \text { if } r \text { not in } \Delta^{\prime},
\end{array}\right.
$$

where $\Delta^{\prime}$ is the free volume $\left(v^{3}-v_{0}\right)^{3}$ per molecule and is a dodeca- 
hedron similar to $\Delta$ but with altitude $\left(a-r_{0}\right) / 2$ instead of $a / 2$. The equation of state may also be obtained.

It would appear that the solution (2) will hold for other types of molecular packing where it is possible to define and estimate a free volume; that is, in those cases where the potential within the cell is uniform and is effectively infinite at the boundary. In the case of cubic packing where one molecule may be considered oscillating at the origin, with the six nearest neighbors regarded as fixed in their mean positions along three axes, the free volume per molecule may easily be shown to be

$$
v_{f}=8\left(v^{3}-r_{0}\right)^{3},
$$

where $v$ is the mean volume per molecule and $r_{0}$ the diameter of the molecule. It is reasonable that Eq. (3) will hold approximately for other kinds of molecular packing with the " 8 " replaced by a different constant.

Thus, one might conclude that the solution (2) would hold for many molecular packings of rigid spheres where it is feasible to define a free volume and where one may have

$$
\varphi(r)=\left\{\begin{array}{cl}
1 / v_{f} & \text { if } r \text { inside } v_{f} \\
0 & \text { if } r \text { outside } v_{f} .
\end{array}\right.
$$

1 William W. Wood, J. Chem. Phys. 20, 1334 (1952).

2 J. G. Kirkwood, J. Chem. Phys. 18, 380 (1950) (1951).

\section{Effect of Opposed Disubstitution on Intensities of Even-Even Electronic Transitions}

\author{
HARDEN MCCONNELI \\ Shell Development Company, Emeryville, California \\ (Received October 7, 1952)
}

$\mathrm{T}$ HE theory of spectroscopic moments devised by Platt $^{1}$ has been used by him for the experimental determination of the even-odd $(g-u)$ character of electronic wave functions of excited singlet states of centrally symmetric molecules. ${ }^{23}$ One feature of this theory is the prediction that the absorption intensities of "forbidden" even-even $(g \rightarrow g)$ electronic transitions in centrally symmetric molecules are not affected by opposed disubstitutions, such as, for example, 2,6-dimethyl substitution in naphthalene. The purpose of this letter is to point out that (a) the above prediction is strictly valid only to within the limits of the Born-Oppenheimer approximation (neglect of perturbations of electronic wave functions by nuclear vibrations) and (b) by inclusion of corrections to this approximation in the spectroscopic moment theory, it is possible to obtain an improved prediction of intensity changes of $g \rightarrow g$ transitions produced by opposed disubstitution in centrally symmetric molecules. Failure of a transition of unknown symmetry to conform at least qualitatively to this new prediction for $g \rightarrow g$ transitions can be taken as evidence that the transition is not $g \rightarrow g$ but $g \rightarrow u$.

The absorption intensity which a $g \rightarrow g$ transition "steals" from a strongly allowed $g \rightarrow u$ transition can be approximated by firstorder perturbation theory. This stolen intensity depends in part on the difference in energy of the $g$ and $u$ excited states. Disubstitution can change this energy difference and thereby change the $g \rightarrow g$ transition intensity. A simplified calculation shows that the equation,

$$
\epsilon=\epsilon_{0}[1-(\Delta \nu / \nu)]^{2},
$$

relates the intensity $\left(\epsilon_{0}\right)$ of the $g \rightarrow g$ transition in the unsubstituted molecule to the intensity $(\epsilon)$ of this transition in the disubstituted molecule. In Eq. (1), $\nu$ is the frequency separating the $g$ and $u$ electronic states, and $\Delta \nu$ is the change of this frequency produced by opposed disubstitution. More detailed expressions relating and $\epsilon_{0}$ can be derived, but for many molecular spectra it appears reasonable that $\nu$ can be taken as the frequency separating a 0,1 vibrational state of the weak transition (whose excited state symmetry is to be determined) and a 0,0 vibrational state of a strong allowed $g \rightarrow u$ transition; $\epsilon$ and $\epsilon_{0}$ in Eq. (1) then apply the intensities of the 0,1 band of the $g \rightarrow g$ transition.

Equation (1) predicts that on opposed disubstitution the intensity of a $g \rightarrow g$ transition will (a) decrease if $\Delta \nu$ is positive, (b) increase if $\Delta \nu$ is negative, or (c) remain practically unaltered if $(\Delta \nu / \nu) \ll 1$. Sufficient experimental data are not available for an adequate test of these predictions. It is noteworthy, however, that the effect of opposed dimethyl substitutions in naphthalene on the intensity of the ${ }^{1} A-{ }^{-1} L_{b}$ transition cannot be accounted for by Eq. (1); this result is consistent with the previously assigned $u$ symmetry of this ${ }^{1} L_{b}$ excited state. ${ }^{1,3,4}$ Also, 9, 10-dimethyl substitution in anthracene decreases the intensity of the ${ }^{1} A-{ }^{1} C_{b}$ transition to an extent compatible with $\mathrm{Eq}$. (1); this result is consistent with the previously assigned $g$ symmetry of the ${ }^{1} C_{b}$ state of anthracene at $45,000 \mathrm{~cm}^{-1}$ and the assumption that the ${ }^{1} C_{b}$ state steals intensity from the nearby strongly allowed ${ }^{1} B_{b}(u)$ state at $39,500 \mathrm{~cm}^{-1.3,4}$ Further discussion of these predictions will be deferred until more data are available.

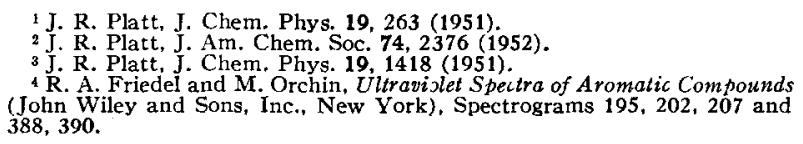
388,390 .

\section{Molecular Weight of Potato Amylopectin as Determined by Light Scattering*}

L. P. Witnauer and F. R. Senti

Eastern Regional Research Laboratory, United States Department of Agriculture, Philadelphia, Pennsylvania

AND

M. D. STERN

Department of Chemistry. Temple University, Philadelphia, Pennsylvania

(Received September 22, 1952)

$\mathbf{M}$ OLECULAR weights reported for amylopectin range from less than 200,000 , as determined by end-group methods, ${ }^{1}$ to 230 million by the light-scattering method. ${ }^{2}$ We report here the results of light scattering measurements on potato amylopectin in water solutions and on acetylated amylopectin in several organic solvents.

Potato starch (potassium form ${ }^{3}$ ) was dispersed with vigorous stirring in water at $90^{\circ}$. The amylose was precipitated with pentanol. Amylopectin recovery was 90 percent. Scattering was measured ${ }^{4,5}$ at angles with 135 to $22^{\circ}$. Reliable extrapolation to $0^{\circ}$ could be made, proving the absence of large particles of dirt or microgel in the solutions. Concentrations ranged from $10^{-4}$ to $10^{-6}$ $\mathrm{g} / \mathrm{ml}$. Solutions were centrifuged at $40,000 \mathrm{rpm}$ and then passed through ultrafine sintered glass filters. Three molecular weight determinations averaged 36 million \pm 10 percent. Values at 546 and $436 \mathrm{mu}$ for a series of solutions of varying concentration agreed to \pm 5 percent. The $90^{\circ}$ scattering was unaffected by temperature in the range 25 to $75^{\circ}$. An amylopectin solution $0.5 \mathrm{~N}$ in sodium hydroxide was heated two hours at $90^{\circ}$. It showed less than 10 percent decrease in turbidity if oxygen was absent. An amylopectin solution was heated one hour at $120^{\circ}$ in a sealed tube free from oxygen without appreciable change in turbidity. These results indicate that if the light-scattering particles are aggregates they are not broken up by temperature or alkali. In acetone solution the acetate of this amylopectin had a molecular weight (corrected for 40 percent acetyl content) of 38 million.

Nine fractions were obtained by adding ethanol to a water solution of this amylopectin containing 0.1 percent sodium chloride. The molecular weight of the fractions, which represented 93 percent of the amylopectin, ranged from 52 to 7 million. Onethird had a molecular weight 48 to 52 million. The weight average for the nine fractions was 36 million. The particle diameter ranged from $4300 \mathrm{~A}$ to $2200 \mathrm{~A}$.

Another potato starch (calcium form ${ }^{3}$ ) was dispersed by autoclaving at $p \mathrm{H} 6.0$, and the amylose was removed by complexing with nitrobenzene and adsorbing on cotton. The molecular weight 